\title{
Roles of Valproic Acid in Improving Radiation Therapy for Glioblastoma: a Review of Literature Focusing on Clinical Evidence
}

\author{
Satoru Ochiai ${ }^{1 *}$, Yoshihito Nomoto ${ }^{2}$, Yasufumi Yamashita ${ }^{1}$, Yui Watanabe ${ }^{2}$, \\ Yutaka Toyomasu ${ }^{2}$, Tomoko Kawamura ${ }^{2}$, Akinori Takada ${ }^{2}$, Noriko $\mathrm{Ii}^{2}$, Shigeki \\ Kobayashi $^{2}$, Hajime Sakuma ${ }^{2}$
}

\begin{abstract}
Glioblastoma (GBM) is the most common and aggressive type of primary brain neoplasm. The current standard therapy for GBM consists of maximal surgical resection within safe limits, followed by radiation therapy (RT) and chemotherapy with temozolomide. Despite advances in treatment, the prognosis of GBM remains poor. Epileptic seizure is one of the most common symptoms in patients with GBM. Valproic acid (VPA), a histone deacetylase inhibitor, is often used as an anti-epileptic drug in patients with brain neoplasms due to its effectiveness and low toxicity profile. Several in vivo and in vitro studies have indicated that VPA has radiosensitizing effects for gliomas and radioprotective influence on normal brain tissue or hippocampal neurons. The results of several retrospective studies have also indicated potential benefit to improve survival of patients with GBM. Moreover, the promising treatment results of a phase 2 trial of concurrent radiation therapy, temozolomide, and VPA for patients with GBM have been recently reported. The use of VPA in patients with GBM has thus recently receiving more attention. In this article, we review the role of VPA in radiation therapy for GBM, focusing on the clinical evidence.
\end{abstract}

Keywords: Valproic acid - anti-epileptic drug - glioblastoma - radiation therapy - chemoradiation therapy - temozolomide

Asian Pac J Cancer Prev, 17 (2), 463-466

\section{Introduction}

Glioblastoma (GBM) is the most common primary brain neoplasm according to the World Health Organization (Louis et al., 2007). It has been reported that it comprises $15 \%$ of all intracranial neoplasms and about half of malignant primary brain and central nervous system tumors (Ostrom et al., 2014). The prognosis of GBM is still poor despite advance in treatment. The standard therapy consists of surgical resection to the extent that is safely feasible, followed by radiation therapy (RT) and chemotherapy with temozolomide (TMZ) (Stupp et al., 2005; Stupp et al., 2009). The median overall survival (OS) is approximately 15 months (Stupp et al., 2005; Oike et al., 2013; Szczepanek et al., 2013), and the five-year survival rate is less than 10\% (Stupp et al., 2009; Oike et al., 2013).

New-onset seizures often represent the first clinical symptom of patients with GBM. It has been reported that $30-40 \%$ of GBM patients initially presented with epileptic seizures (Beaumont et al., 2000; Kerkhof et al., 2013; Berendsen et al., 2015). Although it still remains controversial, epilepsy has been reported to be a favorable factor for survival in GBM (Chaichana et al., 2009; Stark et al., 2012; Rosati et al., 2013; Vecht et al., 2014; Berendsen et al., 2015).

Valproic acid (VPA), a histone deacetylase inhibitor, is a frequently used anti-epileptic drug (AED) in patients with brain neoplasms due to its effectiveness and relatively low toxicity profile (Chateauvieux et al., 2010; Vecht et al., 2014). In vivo and in vitro data suggest the benefit of use VPA combined with radiation therapy for glioma due to radiosensitizing effect for glioma and radioprotective effect for normal brain tissue or hippocampal neurons (Brown et al., 2008; Fu et al., 2010; Shao et al., 2012; Van Nifterik et al., 2012; Oike et al., 2014; Rubner et al., 2014; Zhou et al., 2014; Hosein et al., 2015; Pont et al., 2015; Thotala et al., 2015; Zhou et al., 2015).

In this article, we review the role of VPA in radiation therapy for GBM, focusing on the clinical evidence.

\section{Effects of AED Use for GBM}

Oberndorfer et al. (2005) evaluated the effects of enzyme inducing AED (EI-AED) and non-EI-AED in 
Satoru Ochiai et al

patients with GBM treated with chemotherapeutic agents (Oberndorfer et al., 2005). One hundred and sixty eight GBM patients treated with surgery, RT and chemotherapy were retrospectively analyzed. Patients were separated into three groups: patients without AED ( $n=88)$, patinets with EI-AED $(n=43)$ and patients with non EI-AED $(n=37)$. A significant difference was detected in survival between non-EI-AED (10.8 months) and EI-AED group (13.9 months). Lomustine (CCNU) was the most frequently used chemotherapeutic agent in the study.

Guthrie et al. evaluated the impact of antiepileptic drugs on survival of patients with GBM (Guthrie et al., 2013). They reviewed a cohort of 236 consecutive patients in whom GBM had been diagnosed in their institution, and 210 patients were included the analysis. Among them, 138 patients $(65.7 \%)$ had received no AED and 72 patients (34.3\%) had received some AED. The patients who had received AED had longer survival compared with those who had not received AED (Mantel-Cox log-rank test, $\mathrm{p}<0.001)$.

Barker et al. reported a retrospective analysis of 544 patients with GBM (Barker et al., 2013). In their cohort, 403 patients $(74.1 \%$ ) were taking an AED during RT. The median OS of their entire cohort was 14 months (range, 0 197 months), and that was 13.8 and 13.5 months in patients taking and not taking an AED during RT, respectively $(\mathrm{p}=0.98)$. In the Cox regression analysis, OS was not associated with AED use during RT ( $\mathrm{p}=0.25$, Hazard ratio [HR] 1.13; 95\% confidence interval [CI], 0.92 - 1.33).

Weller et al. reported a post hoc analysis of the European Organization for Research and Treatment of Cancer (EORTC) 26981-22981/National Cancer institute of Canada (NCIC) CE.3 clinical trial which is the landmark trial established the current standard management for GBM (Stupp et al., 2005; Stupp et al., 2009; Weller et al., 2011). They assessed whether AED modulated the effectiveness of chemoradiotherapy (CRT) with TMZ in patients with newly diagnosed GBM. In the CRT with TMZ arm, the outcome of patients receiving an AED at baseline was similar to that of the patients not receiving an AED (overall survival [OS]: $\mathrm{p}=0.89$, HR $0.98,95 \%$ CI; 0.76 - 1.27).

Although these findings indicate that the AED use in the treatment of GBM has potential benefit to improve the outcome of GBM, the benefit of AED remains unclear.

\section{Comparison between VPA and Other AED in the Treatment of GBM}

In the report of EROTC/NCIC TMZ trial for GBM, patients in were divided into 3 groups: patients without AED $(n=175)$, patients with EI-AED only $(n=252)$ and patients with VPA only $(n=97)$ and the OS was compared (Weller et al., 2011). The patients treated with VPA only had a superior survival benefit from CRT (HR 0.39, 95\% CI; 0.24 - 0.63) compared with patients treated with an EI-AED only (HR 0.69, 95\% CI; $0.53-0.90)$ or patients without any AED (HR 0.68, 95\% CI; 0.49 - 0.93). In the CRT with TMZ arm, the median OS was 13.96 months, 14.42 months, 17.35 months for patients without AED, with EI-AED only and with VPA only, respectively.

In the retrospective study by Guthrie et al., of the evaluated 210 patients, 138 patients received no AED, 24 had VPA, 19 had carbamazepine, 20 had phenytoin, and 9 had another AED (Guthrie et al., 2013). Patients treated with VPA had significantly longer survival than those did not received an AED (Mantel-Cox log-rank test 17.506, $\mathrm{p}<0.001$ ), and patients treated with VPA had a significantly longer survival than those who had received other AEDs (Mantel-Cox log-rank test 5.303, p<0.02).

In the retrospective study by Barker et al., of the evaluated 544 patients, $403(74 \%)$ patients were taking an AED during RT, and VPA was used by 29 patients (7\%) (Barker et al., 2013). Among the patients who were taking AED during RT, median OS of patients taking VPA was 16.9 months, compared with 13.6 months in patients using another AED. Multivariable Cox regression analysis revealed that OS was associated with VPA use during RT (HR 0.67, 95\% CI; $0.27-1.07, \mathrm{p}=0.047$ ). On the other hand, Cox regression analysis showed no association of OS with any of the other AED (phenytoin, levetiracetam, carbamazepine or phenobarbital).

These findings indicate that VPA may have a more advantage in the treatment for GBM compared with other AED.

\section{VPA in Treatment for GBM}

Kerkhof et al. conducted a retrospective study to examine the efficacy of VPA given with or without levetiracetam on seizure control and on survival in patients

Table 1. Summery of the effect of VPA on survival

\begin{tabular}{|c|c|c|c|c|c|c|}
\hline Study & No of Pts & $\begin{array}{c}\text { No of Patients } \\
\text { receiving VPA } \\
(\%)\end{array}$ & $\begin{array}{c}\text { Median OS } \\
\text { with VPA (mo) }\end{array}$ & $\begin{array}{c}\text { Median OS } \\
\text { without VPA } \\
(\mathrm{mo})\end{array}$ & $\begin{array}{c}\text { Median OS } \\
\text { without AED } \\
(\mathrm{mo})\end{array}$ & $\begin{array}{c}\text { Median OS } \\
\text { with other AED } \\
(\mathrm{mo})\end{array}$ \\
\hline Weller (2011) & 524 & $97(18.5)$ & 12.8 & & 12.5 & 13.2 \\
\hline Weller $(2011)^{a}$ & 265 & $49(18.5)$ & 17.4 & & 14 & 14.2 \\
\hline Kerkhof $(2013)^{b}$ & 165 & $105(63.6)$ & 15.9 & 14.2 & & \\
\hline Barker (2013) & 531 & $29(5.4)$ & 16.9 & & 13.5 & 13.6 \\
\hline Barker $(2013)^{c}$ & 134 & $12(9.0)$ & 23.9 & 15.1 & & \\
\hline Berendsen $(2015)^{\mathrm{d}}$ & 212 & $55(25.9)$ & 13.8 & 12.7 & & \\
\hline
\end{tabular}

No: number; Pts: patients; OS: overall survival; mo: months; VPA: valproic acid; AED: anti-epileptic drug; a) cohort of patients treated with concurrent chemoradiation therapy with temozolomide; b) cohort of patients treated with concurrent chemoradiation therapy with temozolomide; c) cohort of patients treated with concurrent chemoradiation therapy with temozolomide; d) cohort of epileptic patinets with glioblastoma 
with GBM treated with CRT (Kerkhof et al., 2013). In the report, they performed Cox regression survival analysis on 165 patients receiving CRT with TMZ. In the cohort, 108 patients received VPA for at least 3 months. Patients using VPA in combination with TMZ showed a longer median survival of 69 weeks (95\% CI; 61.7- 67.3) compared with 61 weeks $(95 \% \mathrm{CI} ; 52.5$ - 69.5) in the group without VPA (HR 0.63, 95\% CI; $0.43-0.92, \mathrm{p}=0.016$ ), adjusting for age, extent of resection, and $\mathrm{O}(6)$-DNA methylguaninemethyltransferase promoter methylation status.

In the retrospective study by Barker et al., the benefit of VPA in subgroup of patients who received CRT with TZM, which is the current standard treatment for GBM, were also evaluated (Barker et al., 2013). Patients receiving VPA during CRT had a median OS of 23.9 months, compared with 15.1 months in patients not receiving VPA $(p=0.25)$. Cox regression analysis of patients receiving CRT with TMZ revealed that VPA use during RT was associated with longer OS with borderline significance (HR 0.54, 95\%CI; $-0.09-1.17, \mathrm{p}=0.06)$, independently RTOG RPA class (HR 1.39, 95\% CI; $1.18-1.6, \mathrm{p}=0.02$ ) and seizure history (HR 1.69, 95\% CI; $1.31-2.07, \mathrm{p}=0.007$ ).

Berendsen et al. reported a retrospective analysis to investigate the relationship between survival and epilepsy at presentation in GBM patients (Berendsen et al., 2015). Six hundred and forty seven patients were included in the analysis. Multivariate regression analysis showed that GBM with epilepsy was significantly associated with an increased OS compared with GBM without epilepsy $(p<0.00005)$. On the other hand, they reported that the survival of patients who received VPA $(n=55$; median OS 13.8 months, 95\%CI; 10.1 - 17.4) did not differ significantly from those with seizures who did not received VPA (n=157; median OS 12.7 months, 95\%CI; 10.6 - 14.8, log-rank test $\mathrm{p}=0.55$, crude HR $0.90,95 \% \mathrm{CI} ; 0.62$ - 1.29, $\mathrm{p}=0.55)$. They concluded that epilepsy is an independent prognostic factor for longer survival in GBM and survival is not associated with VPA treatment.

The effect of VPA in these retrospective studies was summarized in Table 1. Although many retrospective studies have suggested a favorable effect of VPA, there seems to be still controversy in the benefit of VPA on OS of GBM patients. The findings of Berendsen et al. seem to be interesting because none of the patients in their cohort without epilepsy received prophylactic AED treatment (Berendsen et al., 2015). On the other hand, in the retrospective study of Baker et al (Barker et al., 2013), of 544 evaluated patients, seizure was noted before the end of RT in 217 (40\%) patients. However, 403 (74\%) patients were taking AED during RT, suggesting that many took AED to prevent seizures. Besides, according to the findings of Weller et al. and Barker et al, the absolute benefit of VPA seems to be larger when VPA is used concurrently used in CRT with TMZ compared RT alone. (Weller et al., 2011; Barker et al., 2013) (Table 1). The study cohort of Berendsen et al. consisted of heterogeneous population, including patients treated with adjuvant RT or TMZ monotherapy, and without adjuvant therapy (Berendsen et al., 2015). These findings may indicate that the concurrent use of VPA during RT, regardless of the presence of epilepsy or seizure as the symptoms of GBM, is essential to improve the outcome of patients of GBM, and the benefit of VPA may be mainly due to radiosensitizing effect for glioma and radioprotective effect for normal tissue as indicated in vivo and in vitro studies (Brown et al., 2008; Fu et al., 2010; Van Nifterik et al., 2012; Shao et al., 2012; Oike et al., 2014; Rubner et al., 2014; Zhou et al., 2014; Hosein et al., 2015; Pont et al., 2015; Thotala et al., 2015; Zhou et al., 2015).

\section{Prospective Trial of VPA in the Treatment of GBM}

Recently, Krause et al. reported the results of a single-arm phase 2 study of concurrent radiation therapy, temozolomide and VPA for patients with GBM (Krauze et al. 2015). Thirty-seven patients with newly diagnosed GBM were enrolled in the study. Patients received VPA, $25 \mathrm{mg} / \mathrm{kg}$ orally, divided into 2 daily doses concurrent with radiation therapy and TMZ. Median overall survival was reported to be 29.6 months (range, 21 - 63.8 months). The most common grade $3 / 4$ toxicities of VPA in conjunction with radiation therapy and TMZ were blood and bone marrow toxicity $(32 \%)$, neurological toxicity $(11 \%)$, and metabolic and laboratory toxicity $(8 \%)$. The survival outcome of the study is promising and the toxicity profile also seems to be well-tolerated.

In summary, we reviewed the role of VPA in RT for GBM, focusing on the current clinical evidence. Although many pre-clinical and retrospective data suggest the benefit of VPA combined with CRT for GBM, there still remains controversy. VPA seems to be one of the most promising agents to improve the outcome of GBM, the prospective data is still limited. Further investigations are warranted to evaluate the efficacy and to provide optimal treatment.

\section{Acknowledgements}

Funding to pay the Open Access publication charges for this article was provided by Matsusaka Central Hospital. There is no conflict of interest, grant or any other assistance to be disclosed in any authors.

\section{References}

Barker CA, Bishop AJ, Chang M, et al (2013). Valproic acid use during radiation therapy for glioblastoma associated with improved survival. Int J Radiat Oncol Biol Phys, 86, 504-9.

Beaumont A, Whittle IR (2000). The pathogenesis of tumour associated epilepsy. Acta Neurochir (Wien), 142, 1-15.

Berendsen S, Varkila M, Kroonen J, et al (2015). Prognostic relevance of epilepsy at presentation in glioblastoma patients. Neuro Oncol, [Epub ahead of print]

Brown SL, Kolozsvary A, Liu J, et al (2008). Histone deacetylase inhibitors protect against and mitigate the lethality of totalbody irradiation in mice. Radiat Res, 169, 474-8.

Chaichana KL, Parker SL, Olivi A, et al (2009). Long-term seizure outcomes in adult patients undergoing primary resection of malignant brainastrocytomas. Clinical article. J Neurosurg, 111, 282-92.

Chateauvieux S, Morceau F, Dicato M, et al (2010). Molecular 


\section{Satoru Ochiai et al}

and therapeutic potential and toxicity of valproic acid. $J$ Biomed Biotechnol, 2010, 479364.

Fu J, Shao CJ, Chen FR, et al (2010). Autophagy induced by valproic acid is associated with oxidative stress in glioma cell lines. Neuro Oncol, 12, 328-40.

Guthrie GD, Eljamel S (2013). Impact of particular antiepileptic drugs on the survival of patients with glioblastoma multiforme. J Neurosurg, 118, 859-65.

Hosein AN, Lim YC, Day B, et al (2015). The effect of valproic acid in combination with irradiation and temozolomide on primary human glioblastoma cells. J Neurooncol, 122 263-71.

Kerkhof M, Dielemans JC, van Breemen MS, et al. (2013). Effect of valproic acid on seizure control and on survival in patients with glioblastoma multiforme. Neuro Oncol, 15, 961-7.

Krauze AV, Myrehaug SD, Chang MG, et al (2015). A phase 2 study of concurrent radiation therapy, temozolomide, and the histone deacetylase inhibitor valproic acid for patients with glioblastoma. Int J Radiat Oncol Biol Phys, 92, 986-92.

Louis DN, Ohgaki H, Wiestler OD, et al (2007). The 2007 WHO classification of tumours of the central nervous system. Acta Neuropathol, 114, 97-109.

Oberndorfer S, Piribauer M, Marosi C, et al (2005). P450 enzyme inducing and non-enzyme inducing antiepileptics in glioblastoma patients treated withstandard chemotherapy. J Neurooncol, 72, 255-60.

Oike T, Suzuki Y, Sugawara K, et al (2013). Radiotherapy plus concomitant adjuvant temozolomide for glioblastoma: Japanese mono-institutional results. PLoS One, 8, 78943.

Oike T, Ogiwara H, Amornwichet N, et al (2014). Chromatinregulating proteins as targets for cancer therapy. $J$ Radiat Res, 55, 613-28.

Ostrom QT, Gittleman H, Liao P, et al. (2014). CBTRUS statistical report: primary brain and central nervous system tumors diagnosed in the United States in 2007-2011. Neuro Oncol, 16, 1-63.

Pont LM, Naipal K, Kloezeman JJ, et al. (2015). DNA damage response and anti-apoptotic proteins predict radiosensitization efficacy of HDAC inhibitors SAHA and LBH589 in patient-derived glioblastoma cells. Cancer Lett, 356, 525-35.

Rosati A, Poliani PL, Todeschini A, et al (2013). Glutamine synthetase expression as a valuable marker of epilepsy and longer survival in newly diagnosed glioblastoma multiforme. Neuro Oncol, 15, 618-25.

Rubner Y, Muth C, Strnad A, et al (2014). Fractionated radiotherapy is the main stimulus for the induction of cell death and of $\mathrm{Hsp} 70$ release of p53 mutated glioblastoma cell lines. Radiat Oncol, 9, 89 .

Shao CJ, Wu MW, Chen FR, et al. (2012). Histone deacetylase inhibitor, 2-propylpentanoic acid, increases the chemosensitivity and radiosensitivity of human glioma cell lines in vitro. Chin Med $J, \mathbf{1 2 5}, 4338-43$.

Stark AM, van de Bergh J, Hedderich J, et al (2012). Glioblastoma: clinical characteristics, prognostic factors and survival in 492 patients. Clin Neurol Neurosurg, 114, 840-5.

Stupp R, Mason WP, van den Bent MJ, et al (2005). Radiotherapy plus concomitant and adjuvant temozolomide for glioblastoma. $N$ Engl J Med, 352, 987-96.

Stupp R, Hegi ME, Mason WP, et al (2009). Effects of radiotherapy with concomitant and adjuvant temozolomide versus radiotherapy alone on survival in glioblastoma in a randomised phase III study: 5-year analysis of the EORTCNCIC trial. Lancet Oncol, 10, 459-66.

Szczepanek D, Marchel A, Moskała M, et al. (2013). Efficacy of concomitant and adjuvant temozolomide in glioblastoma treatment. A multicentre randomized study. Neurol
Neurochir Pol, 47, 101-8.

Thotala D, Karvas RM, Engelbach JA, et al (2015). Valproic acid enhances the efficacy of radiation therapy by protecting normal hippocampal neurons and sensitizing malignant glioblastoma cells. Oncotarget, 6, 35004-22.

Van Nifterik KA, Van den Berg J, Slotman BJ, et al (2012). Valproic acid sensitizes human glioma cells for temozolomide and $\gamma$-radiation. $J$ Neurooncol, 107, 61-7.

Vecht CJ, Kerkhof M, Duran-Pena A (2014). Seizure prognosis in brain tumors: new insights and evidence-based management. Oncologist, 19, 751-9.

Weller M, Gorlia T, Cairncross JG, et al (2011). Prolonged survival with valproic acid use in the EORTC/NCIC temozolomide trial for glioblastoma. Neurol, 77, 1156-64.

Zhou Y, Xu Y, Wang H, et al (2014). Histone deacetylase inhibitor, valproic acid, radiosensitizes the $\mathrm{C} 6$ glioma cell line in vitro. Oncol Lett, 7, 203-8.

Zhou Y, Niu J, Li S, et al (2015). Radioprotective effects of valproic acid, a histone deacetylase inhibitor, in the rat brain. Biomed Rep, 3, 63-9. 